\title{
The dynamics of red supergiant winds
}

Anita M.S. Richards, Raymond J. Cohen

JBO, University of Manchester, Macclesfield SK11 6TA, UK.

Malcolm D. Gray

Dept. of Physics, UMIST, PO Box 88, Manchester M60 1QD, UK.

Koji Murakawa, Jeremy A. Yates

Dept. of Physical Sciences, University of Hertfordshire, AL10 9AB, UK.

Marion Szymczak

Torún Centre for Astronomy, ul. Gagarina 11, PL-87100, Poland.

John D. Monnier

Harvard-Smithsonian CfA, MS 42, Cambridge, MA, USA.

Mike R.W. Masheder

School of Physics, University of Bristol, Bristol, BS8 1TS, UK.

Huib J. van Langevelde

JIVE, Postbus 2, 7990 AA Dwingeloo, The Netherlands.

\begin{abstract}
During the red supergiant (RSG) stage of massive star evolution, emission from dust and molecules allows the copious stellar winds to be studied in great detail. This help us understand not only the evolutionary stages of the star (which are highly dependent on mass loss rates), but also the morphology of the eventual supernova remnant. Maser emission from $\mathrm{OH}$ and $\mathrm{H}_{2} \mathrm{O}$ has been mapped with milli-arcsec resolution (using MERLIN and the EVN/global VLBI) around RSG including VY $\mathrm{CMa}$, S Per and VX Sgr. The $\mathrm{H}_{2} \mathrm{O}$ masers originate in clouds accelerating away from the star and $\mathrm{OH}$ mainlines masers interleave the outer parts of the $\mathrm{H}_{2} \mathrm{O}$ maser shell. Zeeman splitting of $\mathrm{OH}$ maser lines reveals the orientation and strength of stellar-centred magnetic fields.
\end{abstract}

\section{Clumpy mass loss}

The inner $\mathrm{H}_{2} \mathrm{O}$ maser shell radius is $\sim 5 R_{*}$, just outside the dust formation zone (Greenhill et al. 1995). The $\mathrm{H}_{2} \mathrm{O}$ masers around $\mathrm{S}$ Per form an almost 

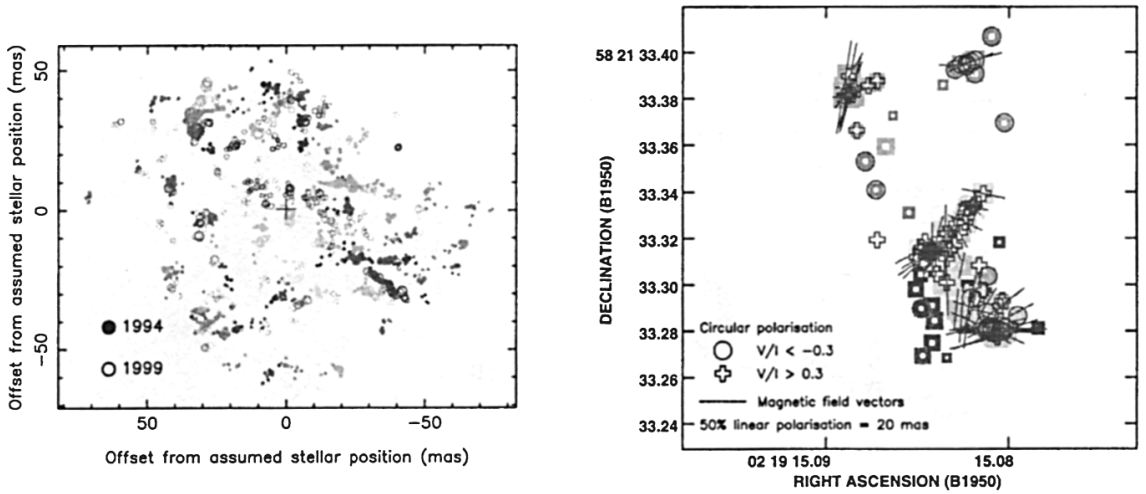

Figure 1. Each spot marks the position of a patch of maser emission from S Per, paler indicating more blue-shifted. (left) $\mathrm{H}_{2} \mathrm{O}$ maser monitoring. (right) $\mathrm{OH} 1665 \mathrm{MHz}$ masers observed in 1999.

spherical shell, shown in Fig. 1(left). The streaks and groups of spots represent emission sampled at $0.1-0.2 \mathrm{~km} \mathrm{~s}^{-1}$ intervals through $\mathrm{H}_{2} \mathrm{O}$ maser clouds.Many of the same clouds can be identified in observations made 5 years apart showing internal turbulence as well as bulk outflow. The clouds are typically 15-20 au in diameter and 50× denser than their surroundings (Richards et al. 1999) at a gas temperature $T_{\mathrm{g}} \sim 1000 \mathrm{~K}$ (Yates et al. 1997). Measurements of proper motions in S Per and VX Sgr (Murakawa priv. comm.) show systematic expansion but no significant rotation.

Fig. 1 (right) shows $\mathrm{OH}$ mainline masers on a similar scale to the $\mathrm{H}_{2} \mathrm{O}$ masers. The $\mathrm{OH}$ shell also overlaps the $\mathrm{H}_{2} \mathrm{O}$ shell in VY CMa and VX Sgr.

1. How is $\mathrm{OH}$ produced in this region, well-shielded from interstellar UV chromospheric radiation? - dissociative shocks from stellar pulations?

2. How are $\mathrm{OH}$ masers pumped at such high densities and temperatures?

The problem is tackled using models including the measured velocity gradients. Fig 2. shows the maser gain for both mainline transitions, based on an $\mathrm{OH}$ cloud size of $10 \mathrm{au}$ and a number density of $3.6 \times 10^{13} \mathrm{~m}^{-3}$. This assumes the $\mathrm{OH}$ masers originate in less dense regions between the $\mathrm{H}_{2} \mathrm{O}$ clouds with a lower dust-gas collision rate at $T_{\mathrm{g}}=400 \mathrm{~K}$. An optical depth of -10 corresponds to an amplification factor of $2.2 \times 10^{5}$; for smaller values the masers are unsaturated, consistent with the observed velocity gradient and variability.

\section{Axisymmetric winds and magnetic fields}

The $\mathrm{H}_{2} \mathrm{O}$ masers are tangentially beamed, indicating acceleration whilst the $\mathrm{OH}$ masers are radially beamed but attain the highest expansion velocities. Fig. 1 shows the $\mathrm{H}_{2} \mathrm{O}$ masers are brightest in regions corresponding to equatorial belts in the near-spherical global distributions, while $\mathrm{OH}$ masers prefer the polar directions; this is typical for RSG. OH masers often show strong Zeeman splitting and some clouds can be $100 \%$ circularly polarised. This can be interpreted by combining the effects of magnetic- and velocity-field gradients in a clumpy medium (Cook 1966). In VX Sgr the OH 1612 masers (Szymczak \& Cohen 1997, Szymczak et al. 2001) indicate a magnetic field of $\sim-1 \mathrm{mG}$ at $\sim 100 R_{*}$. 

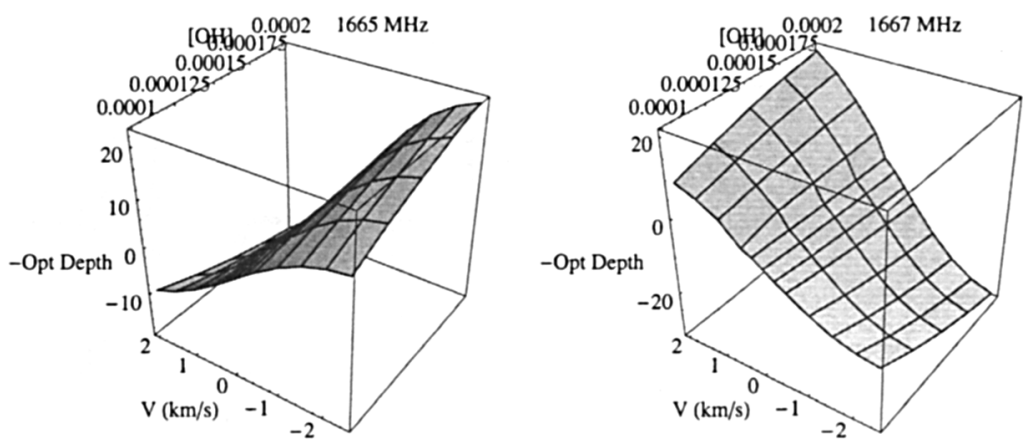

Figure 2. $\quad \mathrm{OH}$ mainline maser gain (negative optical depth) under the conditions in the inner CSE of S Per. $[\mathrm{OH}]$ is the fractional $\mathrm{OH}$ number density and $\mathrm{V}$ is the doppler velocity relative to the cloud centre.

This is supported by EVN observations of $\mathrm{OH}$ mainline masers (Richards et al. 2000 ) and by the polar axis direction deduced from $\mathrm{H}_{2} \mathrm{O}$ and $\mathrm{OH}$ maser velocity field asymmetries. This suggests a dipole field centred on the star at position angle $210^{\circ} \pm 20^{\circ}$, tilted at $\sim 65^{\circ}$ to the plane of the sky. In S Per at $1612 \mathrm{MHz}$ only circular polarisation is found in just two clouds, suggesting a magnetic field pointing towards the observer. Its mainline masers (Fig. 1 right)show the magnetic field is stronger and more complex in regions closer to the star. Linear polarisation is seen in blue-shifted emission only, suggesting Faraday screening of the red-shifted masers by a significant free electron density in the inner CSE.

\subsection{VY CMa}

The $\mathrm{OH}$ masers around VY CMa extend several thousand au from the star in a similar distribution to that of the dust imaged in the NIR by Monnier et al. (1999) (Fig. 3). Irregularities seen in IR emission could originate in the interaction of the stellar wind with orbiting material. The $\mathrm{H}_{2} \mathrm{O}$ masers, in the inner few hundred au, appear to be located in a flared disc, which can also be related to the IR SED (Efstathiou et al. 1990). The $\mathrm{H}_{2} \mathrm{O}$ masers are mostly accelerating strongly away from the star (Richards et al. 1998). However a few clumps show tangential proper motions (on grey background). This may be revealing planetary or protostellar debris.

\section{Conclusions}

We have used radio and IR interferometry to examine the dynamics of stellar winds on scales from tens to thousands of au. The small-scale dynamics of the $\mathrm{H}_{2} \mathrm{O}$ maser region are explained by intrinsically clumpy mass loss from the star. $\mathrm{OH}$ masers are found in between and outside $R_{*}$-sized $\mathrm{H}_{2} \mathrm{O}$-rich clouds, and require different physical conditions. The radially beamed appearance of the $\mathrm{OH}$ may be partly due to the velocity gradients required for maser pumping. The absence or distortion of linearly polarised emission from the far side of 

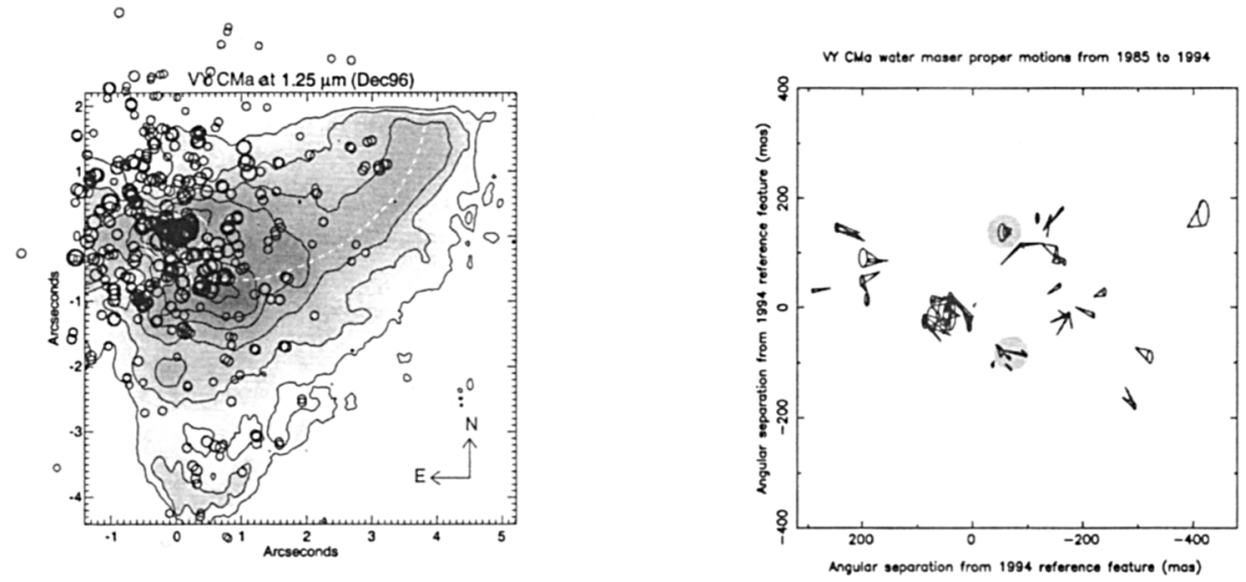

Figure 3. VY CMa: (left) The circles show the positions of $\mathrm{OH}$ mainline maser clouds superimposed on NIR emission (greyscale). (right) Proper motions of $\mathrm{H}_{2} \mathrm{O}$ maser clouds between 1985 and 1993.

the CSE suggests a significant ionisation fraction which may be linked to the production of $\mathrm{OH}$ and other non-LTE chemistry.

Larger-scale asymmetries exist; in VX Sgr and S Per (10-20 solar masses) the $\mathrm{H}_{2} \mathrm{O}$ masers are brightest at velocities close to $V_{*}$ in an equatorial belt. The $\mathrm{OH}$ masers are brightest at more extreme velocities and around the polar axis. This appears to be aligned with a dipole magnetic field in VX Sgr. The magnetic field is strong enough to have a noticeable dynamic influence on the stellar wind and explain the axial symmetries we observe, especially if higher fractional densities of $\mathrm{OH}$ and electrons are associated near the star. VY CMa is more massive and has the only RSG or Mira wind in which we have detected significant (localised) rotation. This may be due to interactions between the outflowing stellar wind and rotating solid material, or other external factors linked to the irregular heart-shaped appearance of the $\mathrm{OH}$ masers and the vast surrounding dusty nebula.

\section{References}

Cook A.H. 1966, Nat., 511, 203

Efstathiou, A., Rowan-Robinson, M. 1990, MNRAS, 245, 275

Greenhill L.J. et al. 1995, ApJ, 481, L23

Monnier et al. 1999, ApJ 512, 351

Richards A.M.S., Yates J.A., \& Cohen R.J. 1998, MNRAS, 299, 319

Richards A.M.S., Yates J.A.,\& Cohen R.J. 1999, MNRAS, 306, 954

Richards A.M.S. et al. 2000, Proc. 5th EVN Symposium, ed. J.E. Conway, A.G.

Polatidis, R.S. Booth \& Y.M. Philström (Onsala Space Observatory), 185

Szymczak M., Cohen R.J., \& Richards A.M.S. 2001 A\&A, 371, 1012

Yates J.A., Field D., \& Gray M.D. 1997 MNRAS, 285, 303 